\title{
Late health effects of ionizing radiation: bridging the experimental and epidemiological divide
}

\author{
P. Jacob $\cdot$ E. Ron
}

Received: 16 February 2010 / Accepted: 16 February 2010 / Published online: 6 March 2010

(C) Springer-Verlag 2010

Knowledge of health risks from chronic or repeated exposures to low-LET radiation with organ doses on the order of $100 \mathrm{mGy}$ or less is of central importance for radiation protection and the safe use of ionizing radiation in medical diagnosis. While it is not yet clear whether the incidence of circulatory diseases is increased after such exposures, there is evidence of an overall elevated risk of cancer. Quantification of site-specific cancer risks and their dependences on exposure patterns, age and other factors still needs clarification. It can be anticipated that approaches to resolve these questions will involve integration of mechanistic and epidemiological methods. The International Conference on Late Health Effects of Ionizing Radiation which was held in Washington, DC, 4-6 May 2009, was organized to stimulate such interdisciplinary research approaches. This volume includes nine papers based on conference presentations.

Recently, radiation-induced genomic instability has been related to cancer incidence in an epidemiological study (Eidemüller et al. 2009). In this volume, Streffer reviews the evidence for the involvement of genomic instability in carcinogenesis and concludes that genetic predisposition for increased radiosensitivity is related to increased genomic instability and cancer predisposition (Streffer 2010). Genomic instability is often assumed to be an early stage in

P. Jacob $(\bowtie)$

Helmholtz Zentrum München,

German Research Center for Environmental Health,

Institute of Radiation Protection, 85764 Neuherberg, Germany

e-mail: jacob@helmholtz-muenchen.de; jacob@gsf.de

E. Ron

Radiation Epidemiology Branch,

National Cancer Institute, NIH, Bethesda,

MD 20892-7238, USA

e-mail: eron@exchange.nih.gov the carcinogenic process. A late step is the progression during which malignant cells develop into clinical symptomatic cancer. In a number of organs, especially thyroid and prostate, dormant or slow-growing tumours often do not develop into clinically relevant tumours. It is an open question how these tumours should be evaluated in assessing cancer risk and latency period. An approach is proposed by Fakir et al. (2010), who address this problem using lung cancer as an example. They developed a mathematical model that takes into account experimental, epidemiological and clinical data to predict latency period and conclude that radiation may be involved in progression from a dormant tumour to a clinically relevant one.

Besides mechanistic understanding, precise dosimetry is important in estimating the health impact of radiation exposures. The large amount of autopsy data from Mayak workers is an important source of information on the biokinetics of inhaled plutonium in airways, the deeper lung, and other organs. In one of the conference presentations, Birchall et al. (2010) described how the Mayak autopsy data on lung tissue clearance and retention rates can impact on current International Commission on Radiological Protection (ICRP) biokinetic models of the respiratory tract. Another area where progress in dosimetry can improve epidemiological studies is computational phantoms. Bolch et al. (2010) review emerging techniques for constructing patientspecific phantoms, which are expected to become the basis of a new generation of dose reconstruction methods.

Recent studies provide some evidence that leukaemia rates are increased after chronic exposures to low-LET radiation with bone marrow doses of approximately 100 $\mathrm{mGy}$. Whether chronic lymphatic leukaemia (CLL) is associated with exposure to ionizing radiation, however, is not clear. Krestinina et al. (2010) present new data on this question. Leukaemia rates among about 30,000 Techa 
River residents chronically exposed to strontium and caesium were analysed. Bone marrow doses, which mainly were due to the incorporation of ${ }^{90} \mathrm{Sr}$, ranged up to $2 \mathrm{~Gy}$ with a median of $0.2 \mathrm{~Gy}$. In this study, CLL was not associated with radiation, but the frequency of other types of leukaemia increased significantly with radiation dose.

Cosmic radiation exposure from air travel is a relatively new area of concern and research because large numbers of crew and passengers are flying frequently. Zeeb et al. (2010) evaluate cancer mortality risk in a cohort of about 30,000 German cockpit and cabin crew members. They found overall cancer mortality risk in the cohort to be similar to that expected based on general population rates. Analyses based on cosmic radiation dose estimates are needed to further assess the elevated SMR for brain cancer found among the cockpit crew. A new mortality follow-up of a large cohort of German uranium miners has added to what is known about radon exposure and subsequent risks of developing specific cancer sites or cardiovascular disease. Kreuzer et al. (2010) report a statistically significantly elevated risk of mortality from cancers of the extra-thoracic airways and trachea, but no association between radon dose and cardiovascular deaths. Another recent focus in radiation research is the better understanding of the role of clastogenic activity. Lindholm et al. (2010) summarize current knowledge about clastogenic plasma factors and suggest ways that these factors could affect health.

Clear indications that circulatory system disease rates are increased after chronic exposures to low-LET radiation with doses on the order of $100 \mathrm{mGy}$ would have considerable impact on the present system of radiation protection. In the past few years, a number of epidemiological studies addressed this question. In this volume, Little et al. (2010) present a review and meta-analysis on this issue. Statistically significant heterogeneity among risk estimates was observed that may be due to confounding or effect modification. Evidence for increased risks of circulatory diseases after exposures with several hundred mGy is increasing. However, evidence after exposures to low-LET radiation at low doses is still missing. Presently, the European Commission funds large mechanistic studies to elucidate possible pathways of radiation effects on the circulatory system (CARDIORISK and NOTE), and an epidemiological study of a large cohort of Mayak workers which has good dosimetry and information on classical risk factors for circulatory diseases, such as smoking, hypertension and obesity (SOLO). It is expected that these studies will improve our understanding of the relation between circulatory diseases and exposures to ionizing radiation, especially at low doses.

\section{References}

Birchall A, Puncher M, Harrison J, Riddell A, Bailey MR, Khokhryakov V, Romanov S (2010) Plutonium worker dosimetry. Radiat Environ Biophys. doi:10.1007/s00411-009-0256-6

Bolch W, Lee C, Wayson M, Johnson P (2010) Hybrid computational phantoms for medical dose reconstruction. Radiat Environ Biophys. doi:10.1007/s00411-009-0260-x

Eidemüller M, Holmberg E, Jacob P, Lundell M, Karlsson P (2009) Breast cancer risk among Swedish hemangioma patients and possible consequences of radiation-induced genomic instability. Mutat Res 669:48-55

Fakir H, Hofmann W, Sachs RK (2010) Modelling progression in radiation-induced lung adenocarcinomas. Radiat Environ Biophys. doi:10.1007/s00411-009-0264-6

Krestinina L, Preston DL, Davis FG, Epifanova S, Ostroumova E, Ron E, Akleyev A (2010) Leukemia incidence among people exposed to chronic radiation from the contaminated Techa River, 19532005. Radiat Environ Biophys. doi:10.1007/s00411-009-0257-5

Kreuzer M, Grosche B, Schnelzer M, Tschense A, Dufey F, Walsh L (2010) Radon and risk of death from cancer and cardiovascular diseases in the German uranium miners cohort study-follow-up 1946-2003. Radiat Environ Biophys. doi:10.1007/s00411-0090249-5

Lindholm C, Acheva A, Salomaa S (2010) Clastogenic plasma factors-a short overview. Radiat Environ Biophys. doi:10.1007/ s00411-009-0259-3

Little MP, Tawn EJ, Tzoulaki I, Wakeford R, Hildebrandt G, Paris F, Tapio S and Elliot P (2010) Review and meta-analysis of epidemiological associations between low/moderate doses of ionizing radiation and circulatory disease risks, and their possible mechanisms. Radiat Environ Biophys. doi:10.1007/s00411-009-0250-z

Streffer C (2010) Strong association between cancer and genomic instability. Radiat Environ Biophys. doi:10.1007/s00411-0090258-4

Zeeb H, Hammer GP, Langner I, Schafft T, Bennack S, Blettner M (2010) Cancer mortality among German aircrew: second followup. Radiat Environ Biophys. doi:10.1007/s00411-009-0248-6 